\title{
Neutrophil Oxygen Radical Generation Synergistic Responses to Tumor Necrosis Factor and Mono/Polyunsaturated Fatty Acids
}

Yongqin Li, ${ }^{\star}$ Antonio Ferrante, ${ }^{\star}$ Alfred Poulos, ${ }^{\ddagger}$ and Dianne P. Harvey*

Departments of*Immunology and ${ }^{\ddagger}$ Chemical Pathology, The Women’s and Children’s Hospital, South Australia 5006, Australia

\begin{abstract}
In inflammatory reactions there are complex interactions of protein mediators (cytokines) and mediators derived from lipids. An important event in inflammation is superoxide production, in relation to microbicidal activity as well as tissue damage. We have studied interactions of lipid mediators with a cytokine mediator tumor necrosis factor alpha (TNF) in stimulating superoxide production by human neutrophils for this reason and because it throws light on intracellular signals activating this response. Pretreatment of neutrophils with TNF markedly augmented the amount of superoxide produced in response to AA but not to either a 20 carbon saturated fatty acid, or the hydroxy- or hydroperoxy-derivatives of AA. Not only were other polyunsaturated fatty acids (eicosapentanoic, docosahexaenoic, linolenic, linoleic acid) as effective as AA but so was the monounsaturated fatty acid, oleic acid. Indeed TNF primed the neutrophils for an increased response to a major mediator of inflammation, leukotriene $\mathrm{B}_{4}$, which is a product of AA metabolism via the lipoxygenase pathway. The data demonstrate that two major types of mediators generated during an inflammatory response have synergistic action on neutrophils in the generation of reactive oxygen species. In contrast, neutrophils primed with TNF and challenged with $\mathrm{PGE}_{2}$, a product of AA metabolism via the cyclooxygenase pathway, showed a reduced chemiluminescence response. This identifies an important interaction between unsaturated lipids and cytokines which is likely to play a critical role in disease processes and nutrient modulation of the immune responses. (J. Clin. Invest. 1996. 97:1605-1609.) Key words: arachidonic acids • cytokines • leukocytes • n-3 fatty acids
\end{abstract}

\section{Introduction}

The initiation, development, duration, and resolution of the inflammatory response is highly dependent on a heterogeneous group of mediators which are produced both at the local tissue as it undergoes "irritation" and damage and by the infiltrating leukocytes (1). Identification of these mediators and understanding their role in inflammation form the basis of past and

Address correspondence to Antonio Ferrante, Department of Immunology, The Women's and Children's Hospital, South Australia 5006, Australia. Phone: 08-204-7216; FAX: 08-204-6046; E-mail: aferrant@ache.mad.adelaide.edu.au

Received for publication 22 September 1995 and accepted in revised form 9 January 1996.

J. Clin. Invest.

(C) The American Society for Clinical Investigation, Inc.

0021-9738/96/04/1605/05 \$2.00

Volume 97, Number 7, April 1996, 1605-1609 present approaches to the development of drugs for immunopharmacological manipulation of inflammation in human diseases. Two different types of mediators which have attracted major interest in this respect are cytokines and fatty acids such as AA and products of its metabolism (1-3). There is at present no appreciation of the relationship between these mediators with respect to their ability to modify each others effects on leukocytes functional responses. In addition, diet manipulation involving different relative quantities of mono and polyunsaturated fatty acids have continued to attract major interest because of their potential use in the management of a range of human diseases (3, 4-9), including rheumatoid arthritis, systemic lupus erythematosus, and cardiovascular diseases, diseases in which TNF is perceived to play a pathophysiologic role (2).

In some types of inflammations, especially those associated with infections, recruitment and activation of neutrophils, with production of superoxide, contribute to tissue damage. Indeed the interaction of superoxide with nitric oxide results in the production of the powerful oxidant peroxynitrite. The finding of specific antibodies to nitrotyrosine in the lungs of humans who have died of respiratory distress syndromes and pneumonia supports the concept that superoxide plays a role in pathogenesis. Furthermore oxidants can activate phospholipase $\mathrm{A}_{2}$ and release AA (10) such that a cascade can occur. In this investigation we have examined the relationship between cytokines and AA by using TNF, a cytokine which has drawn immense interest as a mediator of pathophysiology in infection, cancer, and autoimmune inflammatory diseases (2). Their effects on the neutrophil oxygen radical production was assessed. The studies were extended to include other fatty acids (such as n-3 polyunsaturated fatty acid [PUFA] $)^{1}$ which have formed the basis for diet manipulation in attempts to alter disease processes.

\section{Methods}

Neutrophils. Neutrophils were prepared from blood of healthy volunteers by the rapid single step method (11). Briefly, whole blood was layered onto a medium of Hypaque-Ficoll $(\mathrm{d}=1.114)$ and centrifuged for $30 \mathrm{~min}$ at room temperature. After centrifugation the neutrophils were recovered from the second leukocyte band. These were washed and resuspended in HBSS. The cells were of $>96 \%$ purity and $>99 \%$ viability.

Chemiluminescence. Chemiluminescence was measured as a standard lucigenin-dependent light emission which, based on previous studies, is a measure of superoxide (12). We have previously demonstrated that this assay measures superoxide dismutase-inhibitable chemiluminescence (12). Indeed measurement of superoxide by the classical cytochrome $\mathrm{C}$ reduction assay is not possible because fatty acids inhibit cytochrome $\mathrm{C}$ reduction (12). In the chemilumines-

1. Abbreviations used in this paper: EPA, eicosapentanoic acid; DHA, docosahexaenoic acid; $\mathrm{LTB}_{4}$, leukotriene $\mathrm{B}_{4}$; PUFA, polyunsaturated fatty acid. 
cence assay the neutrophils $\left(1 \times 10^{6}\right)$ were mixed with the various reagents including lucigenin $(250 \mu \mathrm{M})$ in a total volume of $1 \mathrm{ml}$. The tubes were placed in a water-jacketed $\left(37^{\circ} \mathrm{C}\right)$ luminometer chamber (Model 1251 with MultiUse Software; Bio Orbit Oy, Turku, Finland), and the chemiluminescence was recorded as a rate of production over time. The results were expressed as maximal initial rate in millivolts, unless specified otherwise.

Fatty acids, leukotriene $B_{4}\left(L T B_{4}\right)$ and $P G E_{2}$. Oleic acid, linolenic acid, linoleic acid, arachidic acid, AA, eicosapentanoic acid, and docosahexaenoic acid (DHA) were purchased from Sigma Chemical Co., St Louis, MO. The 15-HPETE and 15-HETE were enzymatically generated and purified as described previously (13). A consistency in solubilization of these fatty acids was achieved by incorporating these into dipalmitoyl phosphatidylcholine-micelles. $\mathrm{LTB}_{4}$ and $\mathrm{PGE}_{2}$ were purchased from Sigma.

Tumor necrosis factor. Recombinant human TNF alpha (TNF) was derived from Escherichia coli and contained a specific activity of $6 \times 10^{7} \mathrm{U} / \mathrm{mg}$. This was kindly provided by Dr. G. Adolf (Boehringer Ingelheim, Vienna, Austria).

\section{Results}

Human neutrophils were treated with human recombinant tumor necrosis factor alpha (TNF) for $30 \mathrm{~min}$ and then subjected to stimulation by AA. The response was measured as lucigendependent chemiluminescence, a measure of superoxide (4). Both TNF and AA induced a superoxide response in neutrophils (Table I). However, TNF pretreatment caused a significant and synergistic response to AA (Table I). It was also evi-

Table I. Responses of TNF-primed Neutrophils to Fatty Acids

\begin{tabular}{lc}
\hline Treatment & Chemiluminescence (mV) \\
\hline & mean $\pm S E M$ \\
Vehicle & $0.66 \pm 0.26$ \\
TNF & $9.27 \pm 2.73$ \\
Arachidonic acid & $61.76 \pm 13.15$ \\
TNF + arachidonic acid & $133.45 \pm 43.52^{*}$ \\
Eicosapentaenoic acid & $132.11 \pm 24.29$ \\
TNF + eicosapentaenoic acid & $233.61 \pm 36.99^{*}$ \\
Docosahexaenoic acid & $74.87 \pm 12.24$ \\
TNF + docosahexaenoic acid & $214.88 \pm 41.27^{\ddagger}$ \\
Arachidic acid & $0.82 \pm 0.39$ \\
TNF + arachidic acid & $12.21 \pm 4.36$ \\
Oleic acid & $22.73 \pm 6.66$ \\
TNF + oleic acid & $64.59 \pm 11.58^{\ddagger}$ \\
Linoleic acid & $88.58 \pm 12.38$ \\
TNF + linoleic acid & $239.18 \pm 44.44^{*}$ \\
Linolenic acid & $76.99 \pm 19.81$ \\
TNF + linolenic acid & $218.35 \pm 49.06^{*}$ \\
Hydroxyeicosatetraenoid acid & $4.08 \pm 1.63$ \\
TNF + hydroxyeicosatetraenoic acid & $16.53 \pm 10.03$ \\
Hydroperoxyeicosatetraenoic acid & $3.95 \pm 2.06$ \\
TNF + hydroperoxyeicosatetraenoic acid & $6.33 \pm 2.13$ \\
&
\end{tabular}

The neutrophils were treated with $100 \mathrm{U}$ TNF for $30 \mathrm{~min}$ at $37^{\circ} \mathrm{C}$ and then tested for a response to the indicated fatty acids $(10 \mu \mathrm{g} / \mathrm{ml})$. The effect of combined TNF + fatty acid treatments were significantly different from the sum of the values of TNF alone plus fatty acid alone. $\left({ }^{*} P<\right.$ $0.05 ;{ }^{\ddagger} P<0.01$ ). The results are presented as mean \pm SEM of 4 to $14 \mathrm{ex}-$ periments each conducted with neutrophils from a different donor. The values represent maximal initial rates of chemiluminescence. dent that TNF did not prime the neutrophils for a response to the saturated equivalent 20 carbon fatty acid, arachidic acid (Table I). Conversion of the AA to its hydroxyl (HETE) and hydroperoxy (HPETE) forms resulted in loss of synergism with TNF (Table I). The methyl ester of AA also showed no synergism with TNF (data not presented).

Since cells may be exposed to varying amounts and types of dietary unsaturated fatty acids, we examined whether these other fatty acids could produce a synergistic neutrophil response with TNF. The data in Table I demonstrate that two of the major components of fish oil, eicosapentanoic acid (EPA) and docosahexaenoic acid (n-3 fatty acids) caused augmented responses in TNF-primed neutrophils. These fatty acids were just as active as AA. Other PUFA, linolenic and linoleic acid, also acted synergistically with TNF to activate neutrophils to release oxygen radicals (Table I), and the monounsaturated fatty acid, oleic acid, showed a fourfold increase in neutrophil stimulation with TNF priming (Table I).

Examination of the rate of superoxide (chemiluminescence) production as a function of time showed that the synergism between TNF and PUFA were seen both as an increase in peak rate of chemiluminescence as well as total generated chemiluminescence (Fig. 1). While AA alone had very little effect at $1 \mu \mathrm{g} / \mathrm{ml}$ on neutrophil oxygen radical production, TNFprimed neutrophils gave a substantial response to this concentration of AA (Fig. 2). The results in Fig. 2 also show that the degree of stimulation by AA of TNF-primed neutrophils continued to increase up to $10 \mu \mathrm{g} / \mathrm{ml}$. Varying the concentration of TNF showed that PUFA-TNF-synergistic neutrophil responses were evident at $1 \mathrm{U} / \mathrm{ml}$ and continued to increase up to $100 \mathrm{U} / \mathrm{ml}$ of cytokine (Fig. 3).

Since prostaglandins and leukotrienes are regarded as major mediators of inflammation, we also examined the effects of TNF on responses to $\mathrm{LTB}_{4}$ and $\mathrm{PGE}_{2}$. Pretreatment of neutrophils with TNF resulted in a significantly $(P<0.01)$ enhanced chemiluminescence response to $\mathrm{LTB}_{4}$ (Fig. 4). In contrast, the

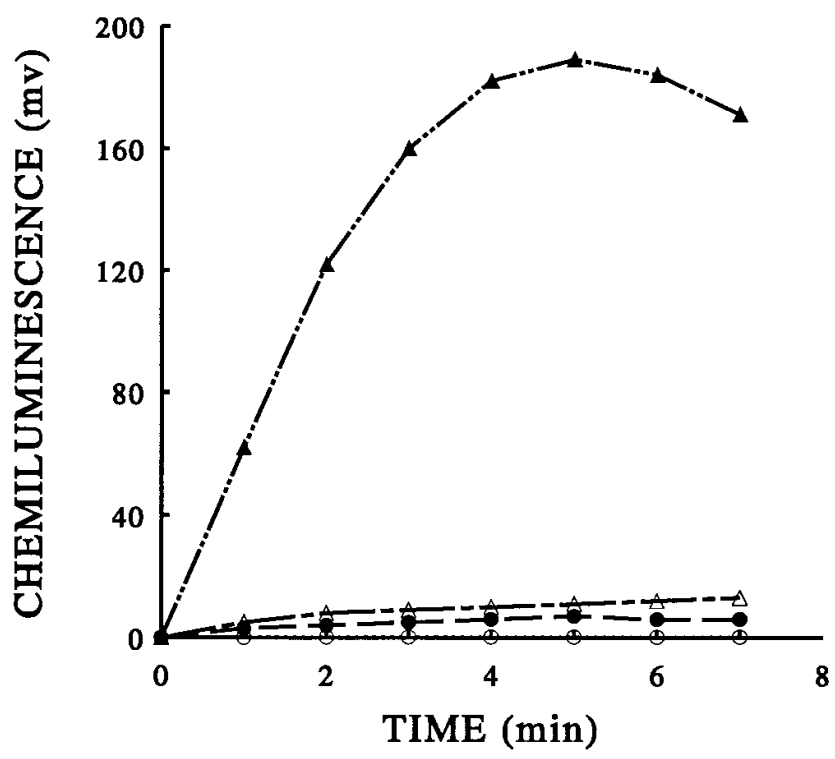

Figure 1. Synergisms between TNF and DHA shown as rate of neutrophil chemiluminescence production over time. Neutrophils were treated with either diluents $(\bigcirc)$, TNF $(100 \mathrm{U})(\bullet)$, DHA $(10 \mu \mathrm{g} / \mathrm{ml})$ $(\triangle)$, or a combination of TNF and DHA $(\Delta)$. Similar results were seen with the other polyunsaturated fatty acids. 


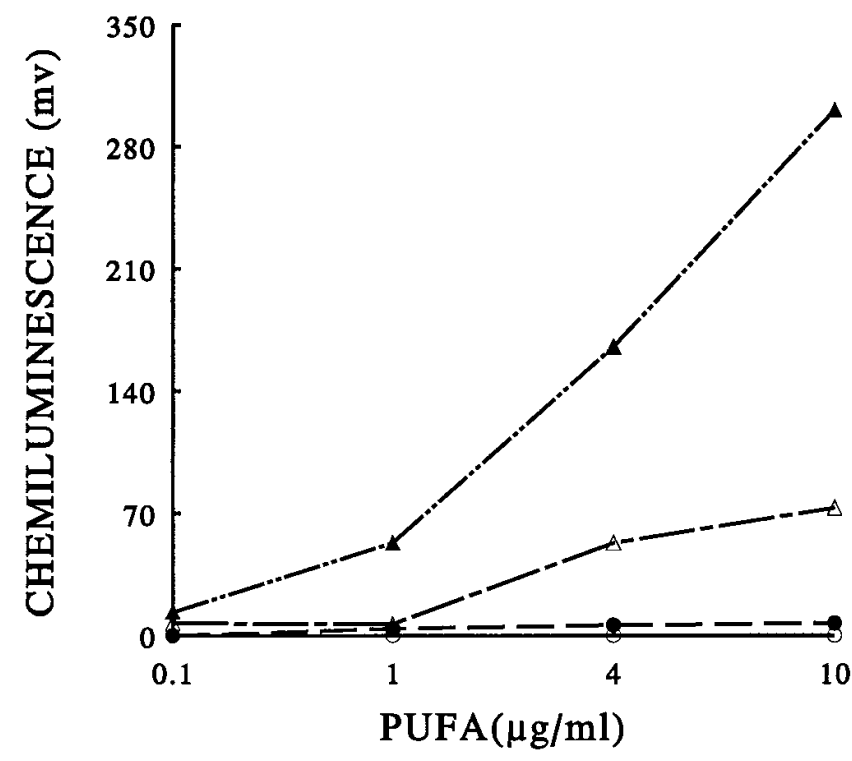

Figure 2. Shows the concentration-related effects of AA on TNFtreated neutrophils on the chemiluminescence response. In these studies neutrophils were pretreated with TNF (100 U) for $30 \mathrm{~min}$ and then challenged with AA. Neutrophils were treated with either diluent $(\bigcirc)$, TNF $(\bullet)$, AA $(\triangle)$, or TNF plus AA $(\mathbf{\Delta})$. Similar results were seen with other PUFAs. Each point represents the maximal initial rate of chemiluminescence.

TNF-treated cells showed a reduced $(\sim 50 \%$ decrease, $P<$ 0.05) chemiluminescence response in the presence of $\mathrm{PGE}_{2}$ (data not presented).

\section{Discussion}

Cytokines are major regulators of the body's inflammatory response. They act on cell surface receptors to induce a range of

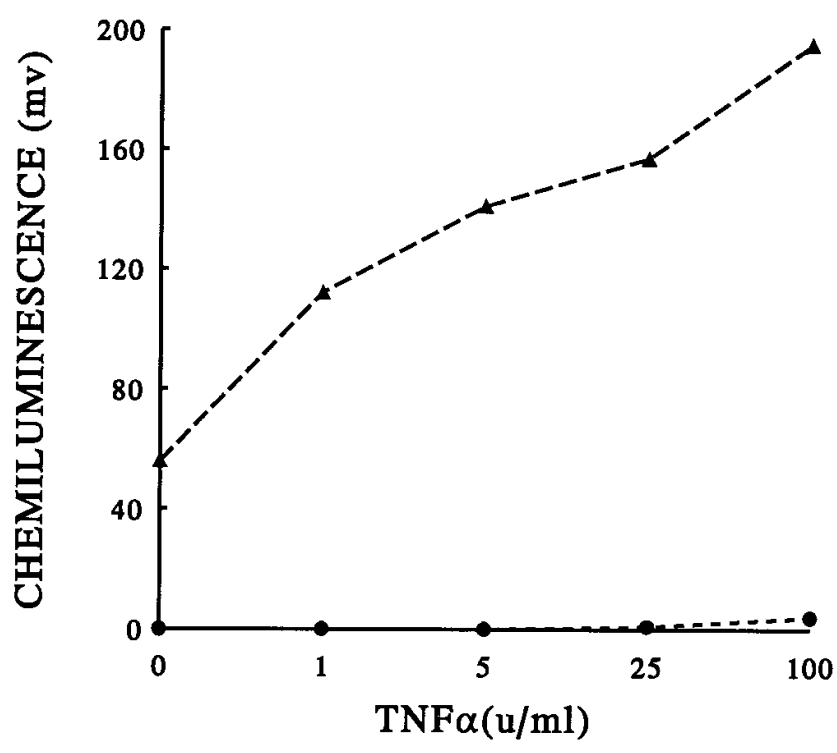

Figure 3. The effects of varying the TNF concentration on the neutrophil response to EPA. Neutrophils were pretreated for $30 \mathrm{~min}$ with the indicated concentrations of TNF and then challenged with either the vehicle $(\bullet)$ or $10 \mu \mathrm{g} / \mathrm{ml}$ of EPA $(\mathbf{\Delta})$. Similar results were seen with the other PUFAs. Each point represents the maximal initial rate of chemiluminescence.

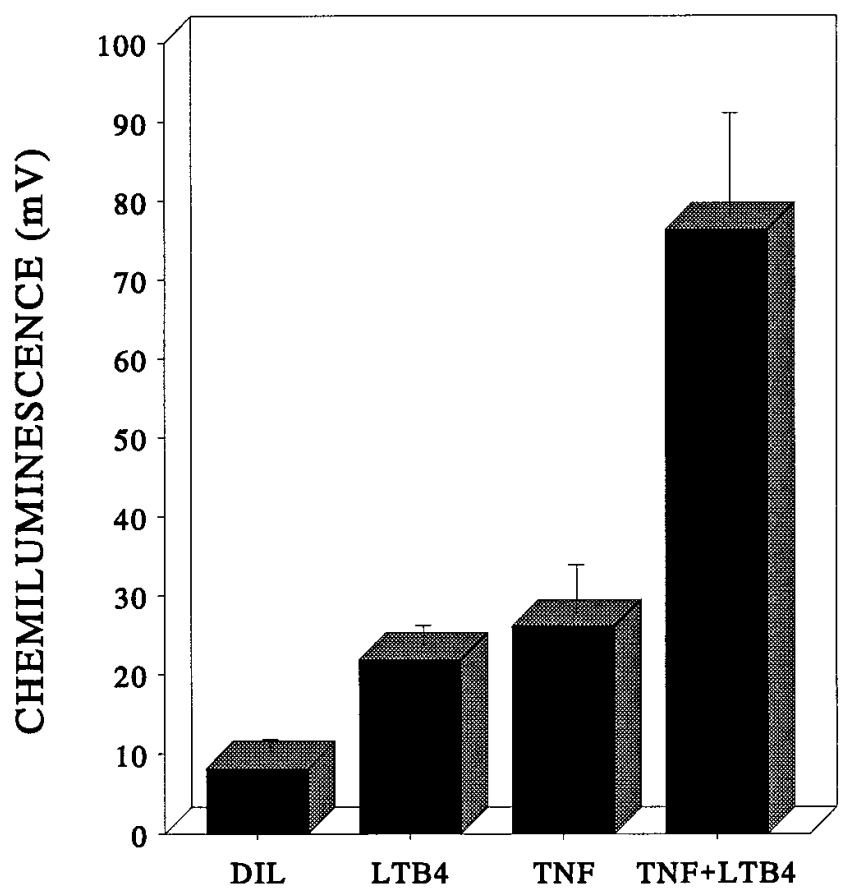

Figure 4. The chemiluminescence response of TNF-primed neutrophils to $\mathrm{LTB}_{4}$. Neutrophils were pretreated with TNF (as described in Table I) and then challenged with $5 \times 10^{-6} \mathrm{LTB}_{4}$. Dil represents the diluent-treated cells (HBSS). The results are the mean \pm SEM of seven experiments.

activities crucial to the characteristics of an inflammatory reaction. TNF is one of the cytokines which has been accredited with the property of altering phagocytic leukocyte activity. A cytokine with pleiotropic effects, TNF, can on the one hand increase resistance against infection and on the other mediate the pathophysiology associated with many diseases (1, 14-21). During cell stimulation, as a result of activation of phospholipase $\mathrm{A}_{2}$, AA is generated. A number of studies have demonstrated that AA has a major influence on leukocyte responses. AA stimulates the production of oxygen radicals (12, 22-26), release of granule enzymes, increases adherence and damage to the endothelium (26-28), and inhibits cell migration (13).

We have now presented evidence that when neutrophils are treated with both TNF and AA, a synergistic superoxide response is seen. This highlights a mechanism by which amplification of the inflammatory response occurs. Production of oxygen radicals by leukocytes is essential for their antimicrobial activity as well as their causation of damage to tissues (1, 29). During infection both TNF and AA are generated, and it is likely that neutrophils become stimulated synergistically by the two types of mediators. In the first instance this may increase the antimicrobial activity of the neutrophil, but, in addition, the tissue-damaging properties of the leukocyte will be potentiated. This phenomenon may also be relevant to tissue damage which occurs in autoimmune inflammatory diseases, such as rheumatoid arthritis.

The synergistic effects of PUFA with TNF were not restricted to the n- 6 fatty acids as the n-3 fatty acids, EPA and DHA, were just as effective. Treatment of neutrophils with both TNF and either EPA or DHA resulted in a marked increase in superoxide production. Thus, any approaches towards manipulation of the host's inflammatory response by di- 
ets rich in the n-3 fatty acids will be limited by the synergistic superoxide production by TNF and the n-3 fatty acids. In many inflammatory diseases, such as arthritis, TNF is produced and associated with the pathogenesis of the disease. Diets rich in n-3 fatty acids used in an attempt to alleviate autoimmune inflammation are likely to act synergistically with endogenously generated TNF in stimulating production of oxygen radicals.

To achieve a synergistic response with TNF, it was evident that fatty acid structure was critical. Long-chain saturated fatty acids lacked activity with respect to this effect. Methylation of the unsaturated long-chain fatty acid also resulted in loss of activity. Enzymatic hydroxylation and peroxidation resulted in a lack of synergistic activity with TNF. Of interest was the finding that linoleic and linolenic acid were as effective as the longchain polyunsaturates. Even the monounsaturated fatty acid, oleic acid, gave rise to a synergistic superoxide response. While the mechanisms of this synergism have not been studied, it is evident that TNF and PUFA stimulate cells via different mechanisms. The PUFAs activate protein kinase $\mathrm{C}$ whereas TNF priming occurs independently of protein kinase $\mathrm{C}$ activation $(21,30,31)$.

Metabolism of AA leads to the release of a variety of eicosanoids, some of which are major regulators of the inflammatory reaction. Alteration of the neutrophil response of these by TNF could be of significance in the inflammatory response. The data presented show that the superoxide production in response to $\mathrm{LTB}_{4}$, a major mediator of inflammation and a product of the lipoxygenase pathway of AA metabolism, was significantly enhanced by TNF. However, there was no synergistic response found between TNF and $\mathrm{PGE}_{2}$, a product of the cyclooxygenase pathway. Indeed $\mathrm{PGE}_{2}$ depressed the TNF-induced superoxide response. These results further point to the importance of the interaction between different mediators in the inflammatory reaction.

Synergistic superoxide responses were seen with TNF concentrations of $1 \mathrm{U} / \mathrm{ml}$ and PUFA concentrations of $1 \mu \mathrm{g} / \mathrm{ml}$. These concentrations are well within the levels attained in vivo during infection, inflammation, and fatty acid diet manipulations. TNF plays an important role in the pathogenesis of many diseases of infectious, malignant, or autoimmune origin, and the cytokine causes myocardial suppression, respiratory arrest, hemorrhagic necrosis, and intravascular coagulopathy (2). Our studies suggest that these effects of TNF may be in part a consequence of the combined action of the cytokine and PUFAs such as AA. Indeed, the limited benefits in using n-3 PUFA and $\gamma$-linolenic in treatment of arthritis (3) may be related to the finding that they cause a synergistic superoxide response in the presence of TNF. While our observations are intriguing, the degree to which they play a role in human therapy remains to be supported by in vivo studies.

\section{Acknowledgments}

We thank Dr. Brenton Robinson for the preparation of the hydroxyand hydroperoxy-derivatives of the parent fatty acids.

This work was supported by grants from the National Health and Medical Research Council of Australia.

\section{References}

1. Gallin, J.I., I.M. Goldstein, and R. Snyderman. 1988. Inflammation: Basic Principles and Clinical Correlates. Raven Press Ltd. New York. 995 pp.

2. Beutler, B. 1992. Tumor Necrosis Factors: the Molecules and their
Emerging Role in Medicine. Raven Press Ltd. New York. 590 pp

3. Cunningham-Rundles, S. 1993. Nutritional modulation of the immune response. Marcel Dekker Inc. New York. 556 pp.

4. Endres, S., R. Ghorbani, V.E. Kelley, K. Georgilis, G. Lonnemann, J.W.M. Van der Meer, J.G. Cannon, T.S. Rogers, M.S. Klempner, P.C. Weber, E.J. Shaefer, S.M. Wolff, and C.A. Dinarello. 1989. The effect of dietary supplementation with n-3 polyunsaturated fatty acids on the synthesis of interleukin-1 and tumor necrosis factor by mononuclear cells. N. Engl. J. Med. 320:265-271.

5. Kremer, J.M., W. Jubiz, A. Michalek, R.I. Rynes, L.E. Bartholomew, R.D. Biganoitte, M. Timchalk, D. Beeler, and L. Liniger. 1987. Fish-oil fatty acid supplementation in active rheumatoid arthritis: a double-blinded, controlled, crossover study. Ann. Intern. Med. 106:497-503.

6. Bittiner, S.B., W.F. Tucker, I. Cartwright, and S.S. Bleehen. 1988. A double blind randomised, placebo-controlled trial of fish oil in psoriasis. Lancet. i: 378-380.

7. Prickett, J.D., D.R. Robinson, and A.D. Steinberg. 1981. Dietary enrichment with the polyunsaturated fatty acid eicosapentaenoic acid prevents proteinuria and prolongs survival in $\mathrm{NZB} \times \mathrm{NZW} \mathrm{F}_{1}$ mice. J. Clin. Invest. 68:556559

8. Kromhout, D., E.B. Bosschieter, and C. deLezenne Coulander. 1985. The inverse relationship between fish consumption and 20-year mortality from coronary heart disease. N. Engl. J. Med. 312:1205-1209.

9. Davis, H.R., R.T. Bridenstine, D. Vesselinovitch, and R.W. Wissler. 1987. Fish oil inhibits development of atherosclerosis in rhesus monkeys. Atherosclerosis. 7:441-449.

10. Robison, T.W., A. Sevanian, and H.J. Forman. 1990. Inhibition of arachidonic acid release by nordidroguaiaretic acid and its antioxidant action in rat alveolar macrophages and Chinese hamster lung fibroblasts. Toxicol. Appl. Pharmacol. 105:112-122.

11. Ferrante, A., and Y.H. Thong. 1980. Optimal conditions for the simultaneous purification of mononuclear and polymorphonuclear leukocytes from human blood by the Hypaque-ficoll method. J. Immunol. Methods. 36:109-117.

12. Hardy, S.J., A. Ferrante, A. Poulos, A. Robinson, D.W Johnson, and A.W. Murray. 1994. The effect of exogenous fatty acids with greater than 22 carbon atoms (very-long-chain fatty acids) on superoxide production by human neutrophils. J. Immunol. 153:1754-1760.

13. Ferrante, A., D.H.B. Goh, D.P. Harvey, B.S. Robinson, C.S.T. Hii, E.J. Bates, S.J. Hardy, D.W. Johnson, and A. Poulos. 1994. Neutrophil migration inhibitory properties of polyunsaturated fatty acids: the role of fatty acid structure, metabolism and possible second messenger systems. J. Clin. Invest. 93: 1063-1070.

14. Ferrante, A. 1992. Activation of neutrophils by interleukin 1 and 2 and tumour necrosis factors. In Granulocyte Responses to Cytokines: Basic and Clinical Research. R.G. Coffey, editor. Marcel Dekker Inc. New York. 417 436 .

15. Ferrante, A., I.C. Kowanko, and E.J. Bates. 1992. Mechanisms of host tissue damage by neutrophils activated by cytokines. In Granulocyte Responses to Cytokines: Basic and Clinical Research. R.G. Coffey, editor. Marcel Dekker Inc. New York. 499-521.

16. Ferrante, A., A.J. Martin, E.J. Bates, D.H.B. Goh, D.P. Harvey, D. Parsons, D. Rathjen, G. Russ, and J.-M. Dayer. 1993. Killing of Staphylococcus aureus by tumour necrosis factor $\alpha$-activated neutrophils: the role of serum opsonins integrin receptors, respiratory burst and degranulation. J. Immunol. 151: 4821-4828.

17. Nathan, C. 1987. Neutrophil activation on biological surfaces. Massive secretion of $\mathrm{H}_{2} \mathrm{O}_{2}$ in response to products of macrophages and lymphocytes. $J$. Clin. Invest. 80:1550-1560.

18. Djeu, J.Y., D.K. Blanchard, D. Halkias, and H. Friedman. 1986. Growth inhibition of Candida Albicans by human polymorphonuclear neutrophils: activation by interferon- $\gamma$ and tumor necrosis factor alpha. Immunology. 137:29802984.

19. Ferrante, A., L.M. Kumaratilake, and D.A Rathjen. 1993. Cytokine regulation of phagocytic cells in immunity to malaria. In Molecular Immunological Consideration in Malaria Vaccine Development. M. Good and A. Saul, editors. CRC Press. 95 pp.

20. Kowanko, I.C., E.J. Bates, and A. Ferrante. 1990. Neutrophil-mediated cartilage injury in vitro is enhanced by tumour necrosis factor alpha. Rheumatol. Int. 10:85-90.

21. Ferrante, A., C.S.T. Hii, and D.A Rathjen. 1995. Positive perspectives on TNFs: promise versus popular purpose. Today's Life Science. 7:40-47.

22. Abramson, S.B., J. Leszynska-Piziak, and G. Weissman. 1991. Arachidonic acid as a second messenger. Interactions with a GTP binding protein of human neutrophils. J. Immunol. 147:231-236.

23. Badwey, J.A., J.T. Curnette, J.M. Robinson, C.B. Berde, M.J. Karnovsky, and M.L. Karnovsky. 1984. Effects of free fatty acids on release of superoxide and on change of shape by human neutrophils. J. Biol. Chem. 259: 7870-7877.

24. Hardy, S.J., B.S. Robinson, A. Poulos, D.P. Harvey, and A.W. Murray. 1991. The neutrophil respiratory burst: responses to fatty acids, fMLP and phorbal ester suggest divergent signalling mechanisms. Eur. J. Biochem. 198: 801-806.

25. Klebanoff, S.J., M.A. Vadas, J.M. Harlan, L.H. Sparks, J.R. Gamble, J.M. Agosti, and A.M. Walterdorph. 1986. Stimulation of neutrophils by tumor 
necrosis factor. J. Immunol. 136:4220-4225.

26. Bates, E.J., A. Ferrante, D.P. Harvey, and A. Poulos. 1993. Polyunsaturated fatty acids increase neutrophil adherence and integrin receptor expression. J. Leukocyte Biol. 53:420-426.

27. Bates, E.J., A. Ferrante, D.P. Harvey, M. Nandoskar, and A. Poulos. 1993. Docosahexanoic acid (22:6,n-3) but not eicosapentaenoic acid (20:5,n3) can induce neutrophil-mediated injury of cultured endothelial cells: an involvement of neutrophil elastase. J. Leukocyte Biol. 54:590-598.

28. Bates, E.J., A. Ferrante, B. Robinson, L. Smithers, and A. Poulos. 1995. Effect of fatty acid structure on neutrophil adhesion, degranulation and damage to endothelial cells. Atherosclerosis. 116:247-259.
29. Klebanoff, S.J. 1988. Phagocytic cells: products of oxygen metabolism. In Inflammation. Basic Principles and Clinical Correlates. J.I. Gallin, I.M. Golstein, and R. Snyderman, editors. Raven Press Ltd. New York. 391-444.

30. Hii, C.S.T., A. Ferrante, Y.S. Edwards, Z.H. Huang, P.J. Hartfield, D.A. Rathjen, A. Poulos, and A.W. Murray. 1995. Activation of mitogen activated protein kinase $\mathrm{C}$ by arachidonic acid in rat liver epithelial WB cells by a protein kinase C dependent mechanism. J. Biol. Chem. 270:4201-4204.

31. Hardy, S.J., A. Ferrante, B.S. Robinson, D.W. Johnson, A. Poulos, K.J. Clark, and A.W. Murray. 1994. In vitro activation of rat brain protein kinase C by polyenoic very-long-chain-fatty acids. J. Neurochem. 62:1546-1551. 